\title{
Comparison of transcriptome between high- and low-marbling fineness in longissimus thoracis muscle of Korean cattle
}

\author{
Seok-Hyeon Beak ${ }^{1}$ and Myunggi Baik ${ }^{1,2, *}$
}

* Corresponding Author: Myunggi Baik Tel: +82-2-880-4809, Fax: +82-2-873-22717,

E-mail: mgbaik@snu.ac.kr

1 Department of Agricultural Biotechnology and Research Institute of Agriculture and Life Sciences, College of Agriculture and Life Science, Seoul National University, Seoul 08826, Korea

2 Institue of Green Bio Science and Technology, Seoul National University, Pyeongchang 25354, Korea

ORCID

Seok-Hyeon Beak

https://orcid.org/0000-0003-4550-5937

Myunggi Baik

https://orcid.org/0000-0001-8373-9772

Submitted Mar 31, 2021; Revised May 4, 2021; Accepted Jun 11, 2021
Objective: This study compared differentially expressed genes (DEGs) between groups with high and low numbers of fine marbling particles (NFMP) in the longissimus thoracis muscle (LT) of Korean cattle to understand the molecular events associated with fine marbling particle formation.

Methods: The size and distribution of marbling particles in the LT were assessed with a computer image analysis method. Based on the NFMP, 10 LT samples were selected and assigned to either high- $(n=5)$ or low- $(n=5)$ NFMP groups. Using RNA sequencing, LT transcriptomic profiles were compared between the high- and low-NFMP groups. DEGs were selected at $\mathrm{p}<0.05$ and $\mid$ fold change $\mid>2$ and subjected to functional annotation.

Results: In total, 328 DEGs were identified, with 207 up-regulated and 121 down-regulated genes in the high-NFMP group. Pathway analysis of these DEGs revealed five significant $(p<0.05)$ Kyoto encyclopedia of genes and genomes pathways; the significant terms included endocytosis $(\mathrm{p}=0.023)$, protein processing in endoplasmic reticulum $(\mathrm{p}=0.019)$, and adipocytokine signaling pathway $(\mathrm{p}=0.024)$, which are thought to regulate adipocyte hypertrophy and hyperplasia. The expression of sirtuin $4(\mathrm{p}<0.001)$ and insulin receptor substrate $2(\mathrm{p}=$ 0.043 ), which are associated with glucose uptake and adipocyte differentiation, was higher in the high-NFMP group than in the low-NFMP group.

Conclusion: Transcriptome differences between the high- and low-NFMP groups suggest that pathways regulating adipocyte hyperplasia and hypertrophy are involved in the marbling fineness of the LT.

Keywords: Adipocytes; Computer Image Analysis; Intramuscular Fat; Korean Native Cattle; Marbling Fineness; Transcriptome

\section{INTRODUCTION}

In beef, intramuscular fat (IMF) or marbling is associated with flavor, juiciness, and tenderness and is a critical factor determining the grade of beef in some countries, including Korea and Japan [1,2]. The IMF content is positively correlated with the beef price [3], and highly marbled beef is a premium consumer product, especially in Korea and Japan [4].

Computer image analysis (CIA) systems have been developed to assess various characteristics of marbling particles (MPs), such as their number, size, and distribution (fineness or coarseness) [5]. We recently reported that the number of fine MPs (NFMP) in the longissimus thoracis muscle (LT) was positively correlated with the auction price of Korean cattle beef [6], indicating that Korean consumers prefer beef with fine MPs (each covering an area of 0.01 to $0.5 \mathrm{~cm}^{2}$ ). The marbling fineness is determined by the marbling fineness index, which is obtained by dividing the NFMP by the ribeye area $\left(\mathrm{cm}^{2}\right)$ and represents the distribution of fine MPs in the LT. A CIA method has been established for measuring fine MPs, but the mechanism of fine MP formation is unknown. 
High-throughput RNA sequencing is a powerful tool for quantifying transcripts [7]. Several RNA sequencing studies have examined the molecular mechanisms of IMF deposition and identified differentially expressed genes (DEGs) between different IMF levels in the LT of cattle [8,9]. The molecular mechanisms responsible for marbling fineness are unknown. Therefore, this study identified DEGs between cattle with high and low NFMP in the LT to understand molecular events associated with formation of fine MPs.

\section{MATERIALS AND METHODS}

\section{Animals}

All experimental procedures followed the Animal Experimental Guidelines of the Seoul National University Institutional Animal Use and Care Committee and were approved by the committee (SNUIACUC: SNU-160408-4). We used LT samples from Korean cattle steers sampled in our previous study [6]. The details of the feeding trial, sampling, and CIA methods can be found in our previous study [6]. Briefly, the size and distribution of MPs were assessed using a CIA method in 43 Korean cattle steers. Of all LT samples, 10 were selected based on the NFMP and assigned to either a high- $(\mathrm{n}=5)$ or low$(n=5)$ NFMP group (Table 1). The NFMP was used as the selection criterion, because it is highly correlated with auction price of beef and is positively correlated with quality grade $\left(\mathrm{R}^{2}=0.41, \mathrm{p}<0.001\right)[6]$. Three samples from each group were randomly selected for RNA sequencing analysis, and all 10 samples were used for quantitative real-time polymerase chain reaction (qPCR) analysis. (Table 1). Each three steers from the high and low group were randomly selected for RNAseq, and total ten steers were used for qPCR to validate the result of RNA-seq.

\section{Carcass grading and the computer image analysis method}

The carcass traits (back fat thickness, ribeye area, marbling score, and quality grade) were graded by trained meat graders according to the Korean carcass grading system of the Korea Institute for Animal Products Quality Evaluation [10]. The carcass auction price was determined by wholesalers after grading in the slaughter house. Detailed methods have been described previously [6].

Cross-sectional photographs of the ribeye area in the LT were taken alongside the 13th thoracic vertebra using HK-333 beef carcass photography equipment (mirror type) (Hayasaka Ricoh, Sapporo, Japan) developed by Kuchida et al [11], and the photographs were analyzed using Beef Analyzer II (Hayasaka Ricoh, Japan), as described previously [6,12]. CIA traits were obtained as described previously [6]. Briefly, the total number of MPs was counted from the binarized fat image. Fine MPs were defined as those covering an area between 0.01 and $0.5 \mathrm{~cm}^{2}$. The fineness index was obtained by dividing the NFMP by the ribeye area $\left(\mathrm{cm}^{2}\right)$.

\section{RNA sequencing}

Total RNA was extracted from LT tissue using TRIzol reagent (Sigma-Aldrich, Milwaukee, WI, USA) in accordance with the manufacturer's protocol. Total RNA was quantified with a ND-2000 spectrophotometer (Thermo Fisher Scientific, Waltham, MA, USA) by recording the absorbance at $260 \mathrm{~nm}$. The RNA quality was checked via agarose gel electrophoresis and ethidium bromide staining of the $28 \mathrm{~S}$ and $18 \mathrm{~S}$ bands, using an Agilent 2100 Bioanalyzer with the RNA

Table 1. Carcass and CIA characteristics of all animal and animals used for RNA sequencing and qPCR in the high and low number of fine marbling particles groups

\begin{tabular}{|c|c|c|c|c|c|c|c|c|c|}
\hline \multirow[b]{2}{*}{ Variables } & \multirow{2}{*}{$\begin{array}{c}\text { All } \\
\text { animals }^{1)} \\
(n=43)\end{array}$} & \multicolumn{4}{|c|}{ Animals for qPCR } & \multicolumn{4}{|c|}{ Animals for RNA sequencing } \\
\hline & & $\begin{array}{l}\text { High NFMP } \\
\quad(n=5)\end{array}$ & $\begin{array}{l}\text { Low NFMP } \\
\quad(n=5)\end{array}$ & SEM & p-value & $\begin{array}{l}\text { High NFMP } \\
\qquad(n=3)\end{array}$ & $\begin{array}{l}\text { Low NFMP } \\
\quad(n=3)\end{array}$ & SEM & p-value \\
\hline Carcass weight (kg) & 425 & 429 & 420 & 10.5 & 0.688 & 437 & 425 & 12.6 & 0.677 \\
\hline Back fat thickness (mm) & 13.6 & 15.4 & 10.2 & 1.64 & 0.116 & 11.0 & 10.7 & 0.79 & 0.859 \\
\hline Rib eye area $\left(\mathrm{cm}^{2}\right)$ & 90.8 & 93.8 & 86.6 & 3.54 & 0.175 & 97.3 & 89.0 & 2.18 & 0.030 \\
\hline Auction price (\#) & 17,593 & 20,938 & 17,706 & 595 & $<0.001$ & 21,102 & 17,538 & 880 & $<0.001$ \\
\hline \multicolumn{10}{|l|}{ CIA traits } \\
\hline Number of MPs & 3,085 & 3,519 & 2,399 & 381 & 0.151 & 4,042 & 2,560 & 602 & 0.257 \\
\hline NFMP & 203 & 282 & 156 & 21.3 & $<0.001$ & 289 & 158 & 29.9 & $<0.001$ \\
\hline Fineness index & 2.37 & 3.13 & 2.03 & 0.20 & $<0.001$ & 3.10 & 1.98 & 0.26 & $<0.001$ \\
\hline
\end{tabular}

1) These data were reported in our previous study [6].

CIA, computer image analysis; qPCR, quantitative real-time polymerase chain reaction; NFMP, number of fine marbling particles; SEM, standard error of mean; MP, marbling particle.

2) Yield index $=68.184-0.625 \times$ back-fat thickness $+0.13 \times$ rib eye area $-0.024 \times$ carcass weight +3.23 .

${ }^{3)}$ Marbling score: $1=\min ; 9=\max$. 
6000 Nano Chip (Agilent Technologies, Santa Clara, CA, USA). RNA samples were stored at $-70^{\circ} \mathrm{C}$ until RNA sequencing and qPCR analyses. The cDNA library was constructed and sequenced at ebiogen (Seoul, Korea). The library was constructed using a SENSE 3' mRNA-Sequencing Library Prep kit (Lexogen, Vienna, Austria) [13] according to the manufacturer's instructions. Briefly, an oligo-dT primer containing an Illumina-compatible sequence at its $5^{\prime}$ end was hybridized with $500 \mathrm{ng}$ of total RNA, and reverse transcription was initiated. After degrading the RNA template, the second strand was synthesized using a random primer containing an Illumina-compatible linker sequence at its $5^{\prime}$ end. To remove all reaction components, the double-stranded library was purified using magnetic beads. The library was then amplified to add the complete adapter sequences required for cluster generation. High-throughput sequencing was conducted as single-end 75-bp sequencing using a NextSeq 500 system (Illumina, San Diego, CA, USA). The quality of the sequencing reads from LT tissues was checked using FastQC. The reads that passed quality control were mapped to the Bos taurus genome (UMD3.1) from the UCSC database using Tophat2 (v2.0.2) and counted using HTSeq (v0.5.3p3).

\section{Identifying and annotating differentially expressed genes}

For data analysis, the SENSE 3' mRNA sequencing reads were aligned using Bowtie2 ver. 2.1.0. [14]. Bowtie2 indices were generated from either the genome assembly sequence or representative transcript sequences for alignment to the genome and transcriptome. The alignment file was used to assemble transcripts, estimate transcript abundances, and detect DEGs. DEGs between the high- and low-NFMP groups were determined based on counts from the unique and multiple alignments. The read count data were processed based on the quantile normalization method using Genowiz ver. 4.0.5.6 (Ocimum Biosolutions, Hyderabad, India). For functional annotation, we selected significant DEGs based on $\mathrm{p}<0.05$ and $\mid$ fold change (FC) $\mid>2$. The DEGs were categorized based on gene ontology (GO) and Kyoto encyclopedia of genes and genomes (KEGG) annotations using the Database for Annotation, Visualization and Integrated Discovery (DAVID) tool (v6.7) (http://david.abcc.ncifcrf.gov/) [15]. The RNA sequencing data obtained here have been submitted to the NCBI Gene Expression Omnibus (GEO) under accession number GSE166206 (http://www.ncbi.nlm.nih.gov/geo/).

\section{Quantitative real-time polymerase chain reaction}

cDNA was synthesized from the RNA templates $(2 \mu \mathrm{g})$ using an iScript cDNA synthesis kit (Bio-Rad Laboratories, Hercules, CA, USA). qPCR was performed as reported elsewhere [16] using QuantiTect SYBR Green RT-PCR Master Mix (QIAGEN, Hilden, Germany). Briefly, qPCR analyses were performed using Rotor-Gene Q (QIAGEN, Germany) in a $25-\mu \mathrm{L}$ total reaction volume. The mixture contained $20 \mathrm{ng}$ of cDNA, $1.25 \mu \mathrm{L}$ of $10 \mu \mathrm{M}$ primers, and $12.5 \mu \mathrm{L}$ of SYBR Green RTPCR Master Mix. The reaction started at $95^{\circ} \mathrm{C}$ for $15 \mathrm{~min}$, followed by 40 cycles of $94^{\circ} \mathrm{C}$ for $15 \mathrm{~s}, 55^{\circ} \mathrm{C}$ for 30 $\mathrm{s}$, and $72^{\circ} \mathrm{C}$ for $30 \mathrm{~s}$. Primer information can be found in Supplementary Table S1. The $\Delta \Delta$ CT method was used to determine relative FC values [17]. qPCR data were normalized relative to the level of the $\beta$-actin housekeeping gene, as validated in our previous study [16].

\section{Statistical analysis}

Statistical differences between the two groups were determined using the independent t-test or Mann-Whitney test depending on data normality with IBM SPSS ver. 22 (SPSS, Chicago, IL, USA).

\section{RESULTS AND DISCUSSION}

Carcass and computer image analysis characteristics of the animals

We compared the carcass and CIA characteristics between the high- and low-NFMP samples used RNA sequencing and qPCR (Table 1). The high-NFMP group had a higher marbling score, NFMP, and fineness index than did the lowNFMP group (all $\mathrm{p}<0.001$ ). The meat from the high-NFMP group also fetched a higher auction price $(\mathrm{p}<0.001)$, confirming the result in our previous report [6].

\section{RNA sequencing and identification of differentially expressed genes}

Six cDNA libraries were constructed, and an average of 20,585,914 single-end 75-bp sequence reads was generated. At least $95.1 \%$ of the reads in each sample were mapped to the bovine reference genome (UMD3.1) using Tophat2 (v2.0.2) (Supplementary Table S2). In total, 328 DEGs were identified $(\mathrm{p}<0.05$ and $|\mathrm{FC}|>2$ ), with 207 up-regulated and 121 downregulated genes in the high-NFMP group (Figure 1). The upand down-regulated genes included 97 and 122 proteincoding genes, respectively. Table 2 lists the top 10 up- and down-regulated genes.

Functional annotation of differentially expressed genes The DAVID tool was used for functional annotation of the 328 DEGs, which were categorized as related to biological process, molecular function, and cellular component (Supplementary Tables S3 to S5). The number of genes associated with each pathway was small (Table 3). Nevertheless, there were significant pathway terms for DEGs, including endocytosis, protein processing in endoplasmic reticulum (ER), and adipocytokine signaling pathway, which are thought to affect adipocyte hypertrophy and hyperplasia. 


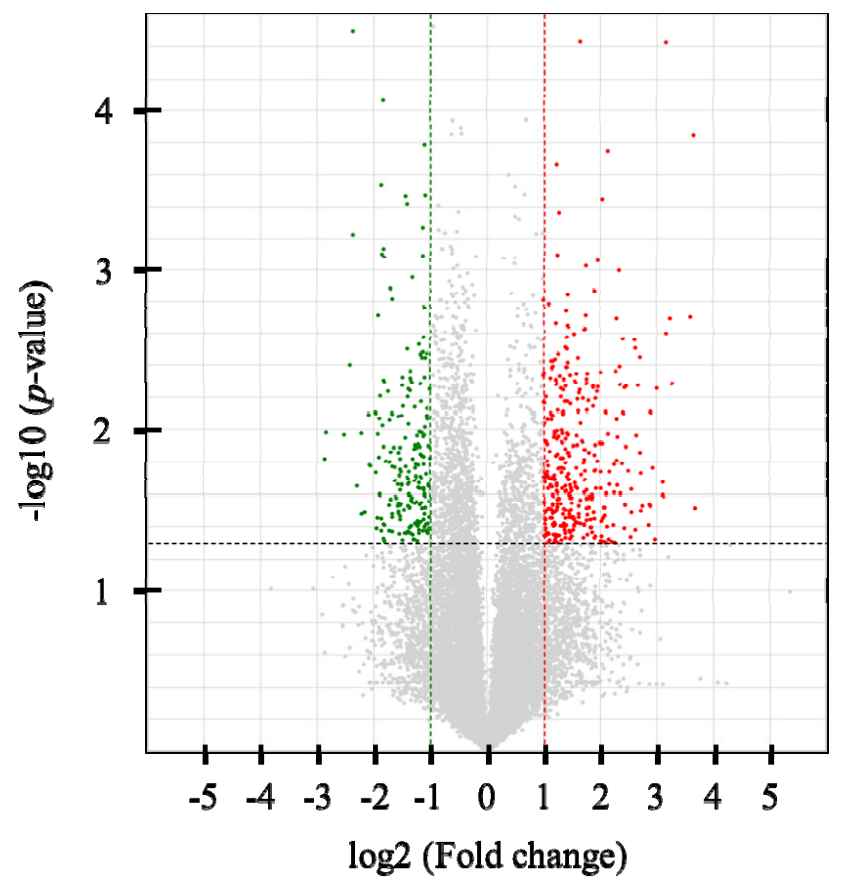

Figure 1. Volcano plot of RNA-sequencing data between the high and low NFMP groups. Up-regulated genes are colored red, and down-regulated genes are colored green in the high NFMP group. Two fold-up and down- regulated threshold lines were shown as dotted vertical red and green line, respectively, and $p$-value $(<0.05)$ threshold line was shown as dotted horizontal line. NEMP, numbers of fine marbling particles.
Eight genes were found to be involved in the endocytosis pathway. Of these, three were up-regulated in the highNFMP group: beta-arrestin-1 (ARRB1; $\mathrm{FC}=2.07, \mathrm{p}=0.019)$, vacuolar protein sorting-associated protein 37D (VPS37D; $\mathrm{FC}=2.54, \mathrm{p}=0.015)$, and ADP-ribosylation factor 3 (ARF3; $\mathrm{FC}=2.35, \mathrm{p}=0.031)$. The other five genes involved in the endocytosis pathway in the high-NFMP group were downregulated: ADP-ribosylation factor 4 (ARF4; $\mathrm{FC}=0.47, \mathrm{p}=$ 0.030), ARF-GAP with coiled-coil, ANK repeat and $\mathrm{PH}$ domain-containing protein $2(A C A P 2 ; \mathrm{FC}=0.31, \mathrm{p}=0.009)$, heat shock $70 \mathrm{kDa}$ protein $1 \mathrm{~B}(H S P A 1 A ; \mathrm{FC}=0.36, \mathrm{p}=0.005)$, heat shock $70 \mathrm{kDa}$ protein $6(H S P A 6 ; \mathrm{FC}=0.24, \mathrm{p}=0.008)$, and vacuolar protein sorting-associated protein 45 (VPS45; $\mathrm{FC}=0.49, \mathrm{p}=0.013$ ). Endocytosis is a vital cellular process in which extracellular ligands, soluble molecules, proteins, and lipids are taken up from the extracellular matrix [18]. Glucose transporter type 4 (GLUT4) is the most abundant glucose transporter regulated by insulin in adipocytes [19]. GLUT4 is translocated from the intracellular matrix to the plasma membrane or vice versa depending on insulin levels; this is orchestrated by exocytosis and endocytosis [20]. Both GLUT4 and CD36, an essential protein for fatty acid endocytosis and uptake in adipocytes, have similar trafficking and recycling systems induced by insulin [21]. Endocytosis is a crucial cellular process through which the uptake of glucose and fatty acid occurs. Through lipogenesis, adipocytes convert glucose into long-chain fatty acids and triglycerides, and finally store lipids as lipid droplets (LDs) [22]. Endocy-

Table 2. Top 10 up and down-regulated genes in the high number of fine marbling particles group

\begin{tabular}{|c|c|c|c|c|}
\hline Order & Gene name & Gene symbol & $\begin{array}{l}\text { Fold change } \\
\text { (high/low) }\end{array}$ & $\mathrm{p}$-value \\
\hline \multicolumn{5}{|c|}{ Up-regulated } \\
\hline 1 & ATCAY kinesin light chain interacting caytaxin & ATCAY & 9.71 & 0.005 \\
\hline 2 & Solute carrier family 28 member 1 & SLC28A1 & 9.45 & 0.002 \\
\hline 3 & BCL2 like 14 & BCL2L14 & 8.98 & 0.003 \\
\hline 4 & TP53 target 5 & TP53TG5 & 6.14 & 0.003 \\
\hline 5 & DEPP1 autophagy regulator & DEPP1 & 5.90 & 0.024 \\
\hline 6 & ATP binding cassette subfamily $\mathrm{C}$ member 6 & ABCC6 & 5.79 & 0.046 \\
\hline 7 & Ankyrin repeat domain 13B & ANKRD13B & 5.39 & 0.029 \\
\hline 8 & MAD2L1 binding protein & MAD2L1BP & 5.36 & 0.041 \\
\hline 9 & Small G protein signaling modulator 2 & SGSM2 & 5.30 & 0.003 \\
\hline 10 & Serine protease 12 & PRSS12 & 5.16 & 0.025 \\
\hline \multicolumn{5}{|c|}{ Down-regulated } \\
\hline 1 & Heat shock protein family A (Hsp70) member 6 & HSPA6 & 0.24 & 0.008 \\
\hline 2 & SHC binding and spindle associated 1 like & SHCBP1L & 0.27 & 0.009 \\
\hline 3 & Peroxisomal biogenesis factor 13 & PEX13 & 0.27 & 0.036 \\
\hline 4 & Proteasome $26 \mathrm{~S}$ subunit, non-ATPase 5 & PSMD5 & 0.28 & 0.010 \\
\hline 5 & DTW domain containing 2 & DTWD2 & 0.28 & $<0.001$ \\
\hline 6 & Leucine rich repeat neuronal 4 & LRRN4 & 0.28 & 0.047 \\
\hline 7 & Hepsin & HPN & 0.28 & 0.001 \\
\hline 8 & Chromosome 7 open reading frame 57 & C4H7orf57 & 0.30 & 0.042 \\
\hline 9 & Cytokine inducible SH2 containing protein & $\mathrm{ClSH}$ & 0.30 & 0.030 \\
\hline 10 & ArfGAP with coiled-coil, ankyrin repeat and PH domains 2 & ACAP2 & 0.31 & 0.009 \\
\hline
\end{tabular}


Table 3. Kyoto encyclopedia of genes and genomes pathway terms for differentially expressed genes

\begin{tabular}{lccl}
\hline Term & Count & p-value & Genes \\
\hline Legionellosis & 5 & $<0.001$ & GR01, CXCL3, HSPA1A, HSPA6, ITGAM \\
Protein processing in endoplasmic reticulum & 5 & 0.019 & DNAJA1, DNAJB1, ER01B, HSPA1A, HSPA6 \\
Endocytosis & 8 & 0.023 & ARF3, ARF4, ACAP2, VPS37D, ARRB1, HSPA1A, HSPA6, VPS45 \\
Butanoate metabolism & 3 & 0.027 & BDH1, AACS, ACSM2B \\
Adipocytokine signaling pathway & 4 & 0.027 & ACSBG2, PRKAG2, LEPR, SLC2A1 \\
Glycerophospholipid metabolism & 4 & 0.059 & CDS1, CRLS1, ETNK2, GPD1L \\
MAPK signaling pathway & 5 & 0.083 & ARRB1, CACNB4, GADD45A, HSPATA, HSPA6 \\
Toxoplasmosis & 4 & 0.084 & ALOX5, HSPA1A, HSPA6 \\
\hline
\end{tabular}

tosis related to GLUT4 and CD36 trafficking may lead to enlargement of the adipocytes (hypertrophy), which may in turn induce adjoining preadipocytes to proliferate (hyperplasia) by secreting paracrine growth factors [23]. Therefore, endocytosis may directly or indirectly affect marbling fineness.

All five DEGs involved in protein processing in the ER pathway were down-regulated in the high-NFMP group: DNAJ homolog subfamily A member 1 (DNAJA1; FC $=0.48$, $\mathrm{p}=0.003$ ), DNAJ homolog subfamily B member 1 (DNAJB1; $\mathrm{FC}=0.48, \mathrm{p}=0.011$ ), endoplasmic reticulum oxidoreductase 1 beta (ERO1B; $\mathrm{FC}=0.46, \mathrm{p}=0.042$ ), HSPA1A, and HSPA6. The ER is a critical cellular organelle controlling calcium homeostasis, lipid synthesis, protein folding, and LD formation, and the size and number of LDs can be affected by the ER stress response [24]. The protein-folding or export capacity of the ER can be exceeded when the ER is stressed under some physiological and pathological conditions [25]. Several studies have reported LD accumulation [26], reduced adipocyte insulin sensitivity [27], and adiponectin oligomerization [28] during the ER stress response. Therefore, the involvement of ER protein processing in adipocyte hyperplasia and hypertrophy may contribute to marbling fineness.

All four DEGs involved in the adipocytokine signaling pathway were up-regulated in the high-NFMP group: long chain fatty acid CoA ligase ACSBG2 isoform X1 (ACSBG2; $\mathrm{FC}=4.91, \mathrm{p}=0.002), 5^{\prime}$-AMP-activated protein kinase subunit gamma-2 isoform X1 (PRKAG2; $\mathrm{FC}=2.15, \mathrm{p}=0.048$ ), leptin receptor (LEPR; $\mathrm{FC}=3.70, \mathrm{p}=0.007$ ), and solute carrier family 2, facilitated glucose transporter member 1 (SLC2A1 or GLUT1; FC = 2.72, p = 0.019). Leptin is mainly secreted by mature adipocytes [29], and high adiposity and body fat increased the concentration and expression of leptin in cattle [30]. Leptin also can induce LD formation in several cell types in an mammalian target of rapamycindependent manner [31,32]. Genetically modified mice without the leptin receptor in adipose tissue were lighter than controls [33], and bone marrow stromal cells with impaired leptin receptors did not differentiate into adipocytes [34]. These results suggest that leptin can directly affect the development and function of adipocytes via leptin receptors. SLC2A1 is a class I glucose transporter that is expressed in adipose tissue at moderate levels [35]. PRKAG2 is a subunit of $5^{\prime}$-AMPactivated protein kinase [36]. Collectively, the adipocytokine signaling pathway may affect adipocyte hyperplasia or hypertrophy, possibly contributing to marbling fineness.

\section{Validation of differentially expressed genes using quantitative real-time polymerase chain reaction} To identify candidate genes associated with marbling fineness, we selected 10 DEGs which may be related with adipocyte hyperplasia and hypertrophy, although these were not listed in the top 10 up- and down-regulated genes shown in Table 2. Description of gene function in UniProt website was considered as selection standard (The UniProt Consortium [37]). The qPCR results were consistent with those from the RNA sequencing analysis for all genes tested, although statistical significance was not obtained in the qPCR analysis (Table 4).

We found higher sirtuin4 (SIRT4) expression in the highNFMP group. Sirtuins are NAD+ dependent deacylases and ADP ribosyltransferases involved in cellular metabolism [38], and SIRT4 is a mammalian sirtuin (SIRT3, SIRT4, and SIRT5) located in mitochondria [39]. SIRT4 plays important roles in insulin secretion, glucose homeostasis [40], and lipid homeostasis, including fatty acid oxidation and lipid anabolism [41]. SIRT4 is highly expressed in bovine subcutaneous adipose tissue and regulates adipogenic differentiationrelated marker genes, including transcription factor E2F1 and CCAAT/enhancer-binding protein $\beta$, during bovine adipocyte differentiation [40]. Overall, the up-regulation of SIRT4 expression may induce adipocyte differentiation, possibly contributing to increasing the NFMP.

We observed higher insulin receptor substrate 2 (IRS2) transcript levels in the LT samples from the high-NFMP group. IRS2 plays an important role in the metabolic activities of insulin and IGF1 in the liver, muscle, and adipose tissue [42]. Cell culture studies suggest that IRS2 also plays an important role in adipocyte differentiation [43]. Therefore, the up-regulated IRS2 expression in the high-NFMP group suggests that it is involved in the formation of MPs in the LT.

Several studies identified DEGs by RNA sequencing analysis and reported transcriptome or genes associated with the 
Table 4. Comparison data of RNA sequencing and $\mathrm{qPCR}$ to validate the selected 10 differentially expressed genes between the high and low number of fine marbling particles groups

\begin{tabular}{|c|c|c|c|c|c|}
\hline \multirow{2}{*}{ Gene name } & \multirow{2}{*}{ Gene symbol } & \multicolumn{2}{|l|}{ RNA-seq } & \multicolumn{2}{|l|}{ qPCR } \\
\hline & & Fold change (high/low) & p-value & Fold change (high/low) & p-value \\
\hline Zinc finger protein $354 \mathrm{C}$ & ZNF354C & 0.32 & 0.008 & 0.61 & 0.440 \\
\hline Attractin & ATRN & 0.46 & 0.003 & 0.45 & 0.220 \\
\hline Peroxisomal trans-2-enoyl-CoA reductase & PECR & 0.46 & 0.003 & 0.50 & 0.260 \\
\hline Insulin receptor substrate 2 & IRS2 & 2.05 & 0.043 & 1.64 & 0.150 \\
\hline C1q and TNF related 5 & C1QTNF5 & 2.36 & 0.022 & 1.15 & 0.720 \\
\hline Peroxisome proliferator activated receptor delta & PPARD & 2.41 & 0.003 & 1.60 & 0.370 \\
\hline Transforming growth factor beta induced & TGFBI & 2.42 & $<0.001$ & 1.44 & 0.400 \\
\hline Sirtuin 4 & SIRT4 & 3.11 & $<0.001$ & 1.86 & 0.080 \\
\hline Folliculin interacting protein 2 & FNIP2 & 4.33 & 0.025 & 2.86 & 0.470 \\
\hline Solute carrier family 28 member 1 & SLC28A1 & 9.45 & 0.002 & 1.10 & 0.740 \\
\hline
\end{tabular}

qPCR, quantitative real-time polymerase chain reaction.

The qPCR results were consistent with those from the RNA sequencing analysis for all genes tested, although statistical significance was not obtained in the $\mathrm{qPCR}$ analysis.

marbling score. For example, the DEGs from the contrast between age and marbling score were recently identified by RNA sequencing analysis in longissimus dorsi muscle of Korean cattle steers, and new potential early age markers and many genes for marbling development were found [44]. In this study, we compared DEGs between groups with highand low- NFMP in the LT of Korean cattle, and the NFMP is used for calculation of fineness index. Our study has a novel aspect that we identified DEGs associated with marbling fineness, which is a new concept for marbling score.

\section{CONCLUSION}

This study is a first attempt to understand the molecular events associated with the formation of fine MPs in the LT. Using RNA sequencing and KEGG pathway analyses, we found that three pathways (adipocytokine signaling pathway, endocytosis, and protein processing in the ER) involved with hyperplasia, hypertrophy, and adipocyte differentiation were significantly enriched in the DEGs. The SIRT4 and IRS2 genes, which are involved in adipocyte hyperplasia and hypertrophy, were up-regulated in the high-NFMP group. Our study suggests that pathways regulating adipocyte hyperplasia and hypertrophy are involved in marbling fineness. Further study is required to determine whether the balance between adipocyte hyperplasia and hypertrophy is linked to marbling fineness.

\section{CONFLICT OF INTEREST}

We certify that there is no conflict of interest with any financial organization regarding the material discussed in the manuscript.

\section{FUNDING}

This work was supported by the National Research Foundation of Korea (NRF) funded by the Ministry of Education (2017R1A2B4003207; 2020R1I1A1A01052628).

\section{SUPPLEMENTARY MATERIAL}

Supplementary file is available from: https://doi.org/10.5713/ ab.21.0150

Supplementary Table S1. Primer sequences of selected DEGs for the qPCR

Supplementary Table S2. The number of sequenced reads and percentage of aligned reads

Supplementary Table S3. Significant biological process pathways of gene ontology analysis.

Supplementary Table S4. Significant molecular function pathways of gene ontology analysis

Supplementary Table S5. Significant cellular component pathways of gene ontology analysis

\section{REFERENCES}

1. Jung EY, Hwang YH, Joo ST. The relationship between chemical compositions, meat quality, and palatability of the 10 primal cuts from Hanwoo steer. Korean J Food Sci Anim Resour 2016;36:145-51. https://doi.org/10.5851/kosfa.2016. 36.2.145

2. Park SJ, Beak SH, Jung DJS, et al. Genetic, management, and nutritional factors affecting intramuscular fat deposition in beef cattle - a review. Asian-Australas J Anim Sci 2018;31: 1043-61. https://doi.org/10.5713/ajas.18.0310

3. Eum SH, Park HR, Seo J, Cho SK, Hur SJ, Kim BW. Multiple regression analysis to estimate the unit price of Hanwoo (Bos taurus coreanae) beef. Korean J Food Sci Anim Resour 2017;37:663-9. https://doi.org/10.5851/kosfa.2017.37.5.663 
4. Gotoh T, Joo ST. Characteristics and health benefit of highly marbled Wagyu and Hanwoo beef. Korean J Food Sci Anim Resour 2016;36:709-18. https://doi.org/10.5851/kosfa.2016. 36.6.709

5. Cheng W, Cheng JH, Sun DW, Pu H. Marbling analysis for evaluating meat quality: methods and techniques. Compr Rev Food Sci Food Saf 2015;14:523-35. https://doi.org/10. 1111/1541-4337.12149

6. Beak SH, Park SJ, Fassah DM, et al. Relationships among carcass traits, auction price, and image analysis traits of marbling characteristics in Korean cattle beef. Meat Sci 2021; 171:108268. https://doi.org/10.1016/j.meatsci.2020.108268

7. Wang Z, Gerstein M, Snyder M. RNA-Seq: A revolutionary tool for transcriptomics. Nat Rev Genet 2009;10:57-63. https:// doi.org/10.1038/nrg2484

8. Yamada T. Genetic dissection of marbling trait through integration of mapping and expression profiling. Anim Sci J 2014;85:349-55. https://doi.org/10.1111/asj.12179

9. Na S, Park SJ, Hong SJ, Baik M. Transcriptome changes associated with fat deposition in the longissimus thoracis of Korean cattle following castration. J Anim Physiol Anim Nutr 2020;104:1637-46. https://doi.org/10.1111/jpn.13393

10.Livestock products grading [Internet]. Sejong, Korea: Korea Institute of Animal Products Quality Evaluation (KAPE); c2019 [cited 2021 Feb 13]. Available from: https://www. ekape.or.kr/english/contents/list.do?menuId=menu156582 \&boardInfoNo

11. Kuchida K, Suzuki M, Miyoshi S. Development of photographing equipment for the cross section of carcass and prediction of BMS number by using obtained image from that equipment. Nihon Chikusan Gakkaiho 2001;72:224-31. https://doi. org/10.2508/chikusan.72.8_224

12. Kuchida K, Osawa T, Hori T, Kotaka S, Maruyama S. Evaluation and genetics of carcass cross section of beef carcass by computer image analysis. Nihon Chikusan Gakkaiho 2006; 34:45-52. https://doi.org/10.5924/abgri2000.34.2_45

13. Moll P, Ante M, Seitz A, Reda T. QuantSeq 3' mRNA sequencing for RNA quantification. Nat Methods 2014;11:1-3. https:// doi.org/10.1038/nmeth.f.376

14.Langmead B, Salzberg SL. Fast gapped-read alignment with Bowtie 2. Nat Methods 2012;9:357-9. https://doi.org/10.1038/ nmeth.1923

15.Dennis G, Sherman BT, Hosack DA, et al. DAVID: Database for annotation, visualization, and integrated discovery. Genome Biol 2003;4:R60. https://doi.org/10.1186/gb-2003-4-9-r60

16. Park SJ, Kang HJ, Na S, Lee SH, Baik M. Differential expression of extracellular matrix and integrin genes in the longissimus thoracis between bulls and steers and their association with intramuscular fat contents. Meat Sci 2018;136:35-43. https://doi.org/10.1016/j.meatsci.2017.10.008

17. Livak KJ, Schmittgen TD. Analysis of relative gene expression data using real-time quantitative PCR and the 2(T)(-Delta
Delta C) method. Methods 2001;25:402-8. https://doi.org/ 10.1006/meth.2001.1262

18. Kiss AL, Botos E. Endocytosis via caveolae: alternative pathway with distinct cellular compartments to avoid lysosomal degradation? J Cell Mol Med 2009;13:1228-37. https://doi. org/10.1111/j.1582-4934.2009.00754.x

19. Ducluzeau PH, Fletcher LM, Vidal H, Laville M, Tavare JM. Molecular mechanisms of insulin stimulated glucose uptake in adipocytes. Diabetes Metab 2002;28:85-92.

20. Hou JC, Pessin JE. The Ins (endocytosis) and Outs (exocytosis) of GLUT4 Trafficking. Curr Opin Cell Biol 2007;19:466-73. https://doi.org/10.1016/j.ceb.2007.04.018

21.van Oort MM, van Doorn JM, Bonen A, et al. Insulin-induced translocation of $\mathrm{CD} 36$ to the plasma membrane is reversible and shows similarity to that of GLUT4. Biochim Biophys Acta 2008;1781:61-71. https://doi.org/10.1016/j.bbalip.2007. 11.006

22. Walther TC, Farese RV Jr. Lipid droplets and cellular lipid metabolism. Annu Rev Biochem 2012;81:687-714. https:// doi.org/10.1146/annurev-biochem-061009-102430

23. Mohamed-Ali V, Pinkney JH, Coppack SW. Adipose tissue as an endocrine and paracrine organ. Int J Obes 1998;22: 1145-58. https://doi.org/10.1038/sj.ijo.0800770

24. Hapala I, Marza E, Ferreira T. Is fat so bad? Modulation of endoplasmic reticulum stress by lipid droplet formation. Biol Cell 2011;103:271-85. https://doi.org/10.1042/BC20100144

25. Pineau L, Ferreira T. Lipid-induced ER stress in yeast and $\beta$ cells: parallel trails to a common fate. FEMS Yeast Res 2010; 10:1035-45. https://doi.org/10.1111/j.1567-1364.2010.00674.x

26. Rutkowski JM, Stern JH, Scherer PE. The cell biology of fat expansion. J Cell Biol 2015;208:501-12. https://doi.org/10.1083/ jcb.201409063

27. Xu L, Spinas GA, Niessen M. ER stress in adipocytes inhibits insulin signaling, represses lipolysis, and alters the secretion of adipokines without inhibiting glucose transport. Horm Metab Res 2010;42:643-51. https://doi.org/10.1055/s-00301255034

28. Huh JY, Seo EY, Lee HB, Ha H. Glucose-based peritoneal dialysis solution suppresses adiponectin synthesis through oxidative stress in an experimental model of peritoneal dialysis. Perit Dial Int 2012;32:20-8. https://doi.org/10.3747/ pdi.2009.00228

29. Zhang Y, Proenca R, Maffei M, Barone M, Leopold L, Friedman JM. Positional cloning of the mouse obese gene and its human homologue. Nature 1994;372:425-32. https://doi.org/ $10.1038 / 372425 \mathrm{a} 0$

30. Jung DJS, Baik M. Up-regulation of bone morphogenetic protein and its signaling molecules following castration of bulls and their association with intramuscular fat content in Korean cattle. Sci Rep 2019;9:19807. https://doi.org/10.1038/ s41598-019-56439-2

31. Maya-Monteiro CM, Almeida PE, D’Avila H, et al. Leptin 
induces macrophage lipid body formation by a phosphatidylinositol 3-kinase- and mammalian target of rapamycindependent mechanism. J Biol Chem 2008;283:2203-10. https:// doi.org/10.1074/jbc.M706706200

32. Fazolini NPB, Cruz ALS, Werneck MBF, Viola JPB, MayaMonteiro CM, Bozza PT. Leptin activation of mTOR pathway in intestinal epithelial cell triggers lipid droplet formation, cytokine production and increased cell proliferation. Cell Cycle 2015;14:2667-76. https://doi.org/10.1080/15384101.2 015.1041684

33. Pereira S, O'Dwyer SM, Webber TD, et al. Metabolic effects of leptin receptor knockdown or reconstitution in adipose tissues. Sci Rep 2019;9:3307. https://doi.org/10.1038/s41598019-39498-3

34. Yue R, Zhou BO, Shimada IS, Zhao Z, Morrison SJ. Leptin receptor promotes adipogenesis and reduces osteogenesis by regulating mesenchymal stromal cells in adult bone marrow. Cell Stem Cell 2016;18:782-96. https://doi.org/10.1016/j. stem.2016.02.015

35. Wood IS, Trayhurn P. Glucose transporters (GLUT and SGLT): expanded families of sugar transport proteins. Br J Nutr 2003; 89:3-9. https://doi.org/10.1079/BJN2002763

36. Gao G, Fernandez CS, Stapleton D, et al. Non-catalytic b- and $\mathrm{g}$-subunit isoforms of the '5-AMP-activated protein kinase. J Biol Chem 1996;271:8675-81. https://doi.org/10.1074/jbc. 271.15.8675

37. The UniProt Consortium, UniProt: a worldwide hub of protein knowledge. Nucleic Acids Res 2019;47:D506-15. https://doi. org/10.1093/nar/gky1049
38. Finkel T, Deng CX, Mostoslavsky R. Recent progress in the biology and physiology of sirtuins. Nature 2009;460:587-91. https://doi.org/10.1038/nature08197

39. Houtkooper RH, Pirinen E, Auwerx J. Sirtuins as regulators of metabolism and healthspan. Nat Rev Mol Cell Biol 2012; 13:225-38. https://doi.org/10.1038/nrm3293

40. Hong J, Li S, Wang X, Mei C, Zan L. Study of expression analysis of SIRT4 and the coordinate regulation of bovine adipocyte differentiation by SIRT4 and its transcription factors. Biosci Rep 2018;38: BSR20181705. https://doi.org/ 10.1042/BSR20181705

41. Laurent GL, German NJ, Saha AK, et al. SIRT4 coordinates the balance between lipid synthesis and catabolism by repressing malonyl CoA decarboxylase. Mol Cell 2013;50: 686-98. https://doi.org/10.1016/j.molcel.2013.05.012

42. Miki H, Yamauchi T, Suzuki R, et al. Essential role of insulin receptor substrate 1 (IRS-1) and IRS-2 in adipocyte differentiation. Mol Cell Biol 2001;21:2521-32. https://doi.org/10. 1128/MCB.21.7.2521-2532.2001

43. Groeneveld MP, Brierley GV, Rocha NM, Siddle K, Semple RK. Acute knockdown of the insulin receptor or its substrates Irs1 and 2 in 3T3-L1 adipocytes suppresses adiponectin production. Sci Rep 2016;6:21105. https://doi.org/10.1038/ srep21105

44. Heras-Saldana SL, Chung KY, Kim H, Lim D, Gondro C, van der Werf JHJ. Differential gene expression in longissimus dorsi muscle of Hanwoo Steers-new insight in genes involved in marbling development at younger ages. Genes 2020;11: 1381. https://doi.org/10.3390/genes11111381 